\title{
The interplay between teamwork, clinicians' emotional exhaustion, and clinician-rated patient safety: a longitudinal study
}

\author{
Annalena Welp ${ }^{1}$, Laurenz L. Meier ${ }^{2}$ and Tanja Manser ${ }^{3,4^{*}}$
}

\begin{abstract}
Background: Effectively managing patient safety and clinicians' emotional exhaustion are important goals of healthcare organizations. Previous cross-sectional studies showed that teamwork is associated with both. However, causal relationships between all three constructs have not yet been investigated. Moreover, the role of different dimensions of teamwork in relation to emotional exhaustion and patient safety is unclear. The current study focused on the long-term development of teamwork, emotional exhaustion, and patient safety in interprofessional intensive care teams by exploring causal relationships between these constructs. A secondary objective was to disentangle the effects of interpersonal and cognitive-behavioral teamwork.

Methods: We employed a longitudinal study design. Participants were 2100 nurses and physicians working in 55 intensive care units. They answered an online questionnaire on interpersonal and cognitive-behavioral aspects of teamwork, emotional exhaustion, and patient safety at three time points with a 3-month lag. Data were analyzed with cross-lagged structural equation modeling. We controlled for professional role.
\end{abstract}

Results: Analyses showed that emotional exhaustion had a lagged effect on interpersonal teamwork. Furthermore, interpersonal and cognitive-behavioral teamwork mutually influenced each other. Finally, cognitive-behavioral teamwork predicted clinician-rated patient safety.

Conclusions: The current study shows that the interrelations between teamwork, clinician burnout, and clinician-rated patient safety unfold over time. Interpersonal and cognitive-behavioral teamwork play specific roles in a process leading from clinician emotional exhaustion to decreased clinician-rated patient safety. Emotionally exhausted clinicians are less able to engage in positive interpersonal teamwork, which might set in motion a vicious cycle: negative interpersonal team interactions negatively affect cognitive-behavioral teamwork and vice versa. Ultimately, ineffective cognitive-behavioral teamwork negatively impacts clinicianrated patient safety. Thus, reducing clinician emotional exhaustion is an important prerequisite of managing teamwork and patient safety. From a practical point of view, team-based interventions targeting patient safety are less likely to be effective when clinicians are emotionally exhausted.

Keywords: Healthcare team, Interprofessional teams, Teamwork, Emotional exhaustion, Patient safety, Intensive care

\footnotetext{
* Correspondence: tmanser@ethz.ch

${ }^{3}$ Institute for Patient Safety, University Hospital Bonn, Stiftsplatz 12, 53111

Bonn, Germany

${ }^{4}$ ETH Zurich, Department of Management, Technology and Economics,

Weinbergstrasse 56/58, 8092 Zurich, Switzerland

Full list of author information is available at the end of the article
} 


\section{Background}

In recent years, the significance of effective teamwork for the provision of safe, high-quality care in fast-paced, unpredictable environments such as intensive care has been increasingly recognized [1]. Teamwork generally comprises cognitive, behavioral, and interpersonal processes [2-4]. Interpersonal teamwork such as the quality of collaboration between nurses and physicians is considered the foundation upon which team cognitions and behaviors unfold [2]. While interprofessional teamwork has been shown to contribute to improved patient outcomes in low-acuity care settings, evidence on the role of interprofessional teamwork in intensive care is only beginning to emerge $[5,6]$. Further, cognitive-behavioral teamwork such as the extent to which team members share a representation of care tasks or the ability to communicate about and jointly execute this task have also been shown to be associated with patient safety [7, 8].

Nurses and physicians who are dissatisfied with the quality of teamwork in their unit experience more emotional exhaustion $[9,10]$. In acute care, about one third of clinicians are affected by it [11]. Moreover, emotional exhaustion is associated with patient safety indicators such as errors and adverse events, and should therefore be a particular concern in settings such as intensive care where consequences of errors are more severe [12].

Previous research has investigated cross-sectional relationships between either two of the three concepts. Thus, it is not known how teamwork, clinician emotional exhaustion, and patient safety are causally related $[7,10,13]$. For instance, it is not clear whether effective teamwork prevents clinician emotional exhaustion, or whether emotionally exhausted clinicians possess fewer resources to contribute to effective teamwork. Further, the differential role of interpersonal and cognitive-behavioral teamwork in relation to clinician emotional exhaustion and patient safety is unclear $[14,15]$.

This longitudinal study examines causal effects between interpersonal and cognitive-behavioral teamwork, emotional exhaustion, and patient safety in interprofessional intensive care teams. We propose that interpersonal and cognitive-behavioral teamwork have a positive effect on clinician-rated patient safety, and that they reduce clinician emotional exhaustion. In addition, we assume that clinician emotional exhaustion decreases clinician-rated patient safety. Knowledge of causal relationships will generate valuable knowledge on the management of intensive care teams and for improving clinician and patient outcomes.

\section{Methods}

\section{Participants and procedures}

The study was conducted in intensive care units (ICUs) across all language regions in Switzerland. To investigate causal effects, longitudinal data were collected from medical and nursing staff using an online survey that included three assessments at 3-month intervals. We contacted nursing and medical leaders of each unit, informing them about the purpose of the study and asking them to discuss participation within their teams. Unit leaders of participating units were sent a link to the survey and forwarded it to their team.

Out of the 81 ICUs in Switzerland, 55 chose to participate $(67 \%)$. The ICUs comprised all specialties (medical, surgical, pediatric, and mixed) and were distributed across 48 hospitals. Participants were 2100 nurses and physicians (month 1: $n=1460$; month 4: $n=1007$; month $7: n=807 ; n=493$ clinicians participated at all three measurement occasions). Nurses comprised registered nurses (RNs), registered nurses with specialization in intensive care, registered nurses in training for intensive care specialization, and nurse unit managers. Physicians specialized in intensive care, anesthesia, surgery, pediatrics, internal medicine, or trauma medicine and included resident physicians, senior physicians, and head physicians (see Table 1 for descriptive statistics).

To investigate the potential impact of attrition, differences on study variables were tested between participants who completed the month 7 assessment and participants who dropped out of the study before month 7 (see Table 2). Participants who completed the month 7 assessment were slightly older, had more professional experience and longer tenure. They also reported lower emotional exhaustion. This phenomenon is commonly found in longitudinal studies assessing aspects of work stress and interpreted as dropout of highly stressed individuals. We found no significant group differences for the other variables included in the statistical model. Thus, it is unlikely that changes in the sample structure influenced study results - if anything, the effects of emotional exhaustion might have been more pronounced.

\section{Measures}

Reliability statistics for all measures at all measurement occasions are reported in Table 3.

\section{Teamwork}

Teamwork was assessed with two scales covering cognitive-behavioral and interpersonal aspects of teamwork.

\section{Cognitive-behavioral teamwork}

We measured the cognitive-behavioral aspect of teamwork with the validated German, French, and Italian translations of the nine-item safety organizing scale, which was originally developed as a safety culture scale $[16,17]$. However, it essentially measures aspects of a team's organizing and coordination behaviors and underlying cognitions on the team level. For instance, it covers 
Table 1 Descriptive statistics

Month 1

Unit level

Type of hospital (university/cantonal/regional)

Type of ICU (medical/surgical/pediatric/mixed)

Beds

Patients treated per month

Individual level

N

Gender (male/female)

Professional role (nurse/physician)

Head RNs

Intensive care RNs

RNs in intensive care training

Head physicians

Senior physicians

Resident physicians

Workload in hospital (full time/part time)

Workload in ICU (full time/part time)

Age

Tenure (years in organization)

Tenure (years in ICU)

Professional experience

Cognitive-behavioral teamwork

Interpersonal teamwork

Emotional exhaustion

Clinician-rated patient safety

1460

$75(5.1)$

$116(7.9)$

$70(4.8)$

$85(5.8)$

77 (5.3)
Month 4

Month 7

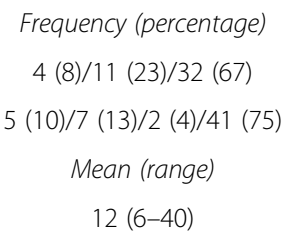

$90(10-405)$

$80(18-314)$

$87(24-447)$

Frequency (percentage)

$1028(70.4) / 365$ (25.0)

1007

807

755 (75.0)/250 (24.8)

$583(72.2) / 218(27.0)$

$1131(77.5) / 243(16.6)$

$506(50.2) / 90(8.9)$

$357(44.2) / 72(8.9)$

$7(0.7)$

$4(0.5)$

$779(53.4)$

114 (11.3)

$82(10.2)$

$16(1.6)$

$20(2.5)$

$7(0.7)$

$7(0.9)$

$25(2.5)$

$25(3.1)$

$684(46.8) / 703$ (48.2)

$20(2.0)$

$33(4.1)$

$443(44.0) / 527$ (52.3)

334 (41.4)/446 (55.3)

$683(46.8) / 700(47.9)$

$443(44.0) / 527$ (52.3)

$201(24.9) / 379(47.0)$

Mean (standard deviation)

$39.56(9.33)$

$9.80(8.35)$

$8.21(7.69)$

$12.57(8.94)$

$5.24(0.81)$

$3.13(0.61)$

$2.73(0.84)$

$3.71(0.62)$
40.44 (9.33)

$40.64(9.07)$

8.29 (7.59)

$7.22(6.71)$

$6.53(7.01)$

$5.35(6.23)$

$11.57(8.71)$

$10.30(8.50)$

$5.25(0.76)$

$5.21(0.78)$

$3.14(0.63)$

$3.11(0.62)$

$2.67(0.83)$

$2.65(0.85)$

$3.71(0.59)$

$3.70(0.59)$

Not all units and participants provided all demographic information at all measurement occasions. $\mathrm{N}=493$ clinicians participated across all three measurement occasions

ICU intensive care unit, $R N$ registered nurse

team cognitions and behaviors such as knowledge about and utilization of collective expertise (sample item: "We have a good map of each other's talents and skills"). Responses are given on a 7-point Likert scale ( $1=$ not at all, 7 = to a very great extent).

\section{Interpersonal teamwork}

We measured the interpersonal aspect of teamwork with the validated German, French, and Italian translations of the three-item nurse-physician relationship scale from the nursing work index revised (NWI-R) [18-20]. The scale assesses clinicians' perception of teamwork quality between nurses and physicians (sample item: "Physicians and nurses have good working relationships"). Responses are given on a 4-point Likert scale $(1$ = disagree, 4 = agree $)$.

\section{Emotional exhaustion}

We measured clinician emotional exhaustion with the validated German, French, and Italian translations [21-23] of the emotional exhaustion subscale of the Maslach Burnout Inventory-Human Services Survey (MBI-HSS) [24]. Emotional exhaustion is the core dimension of burnout, and it is characterized by feeling fatigued, emotionally drained, and lacking the energy to face workrelated tasks (sample item: "I feel mentally exhausted because of my work") [25]. Responses are given on a 6-point Likert scale $(1=$ never, $6=$ very often $){ }^{1}$ 
Table 2 Comparison of participants of month 1 and/or month 4 to participants of all three measurement occasions

\begin{tabular}{|c|c|c|c|c|c|c|}
\hline \multirow{3}{*}{ Gender (male/female) } & \multicolumn{2}{|c|}{ Month 1 and/or 4} & \multicolumn{2}{|c|}{ Month 1, 4, 7} & \multirow{3}{*}{$\begin{array}{l}d f \\
1\end{array}$} & \multirow{3}{*}{$\begin{array}{l}\text { Chi square } \\
0.43\end{array}$} \\
\hline & \multicolumn{3}{|c|}{ Frequency } & & & \\
\hline & \multicolumn{2}{|c|}{$1280 / 278$} & \multicolumn{2}{|c|}{$287 / 102$} & & \\
\hline \multirow[t]{2}{*}{ Professional role } & \multicolumn{2}{|c|}{$1271 / 441$} & \multicolumn{2}{|c|}{$159 / 18$} & 1 & $0.71^{* *}$ \\
\hline & M & SD & M & SD & $d f$ & t value \\
\hline Age & 38.80 & 9.44 & 41.03 & 9.02 & 1408 & $-4.12^{* * *}$ \\
\hline Tenure (years in organization) & 9.00 & 8.05 & 11.04 & 8.76 & 1404 & $-4.16^{* * *}$ \\
\hline Tenure (years in ICU) & 7.30 & 7.44 & 9.69 & 7.87 & 1408 & $-5.29^{* * *}$ \\
\hline Professional experience & 11.62 & 8.99 & 14.16 & 8.51 & 1418 & $-4.84^{* * *}$ \\
\hline Cognitive-behavioral teamwork month 1 & 5.23 & 0.83 & 5.27 & 0.77 & 1347 & -0.87 \\
\hline Interpersonal teamwork month 1 & 3.11 & 0.63 & 3.14 & 0.59 & 1332 & -0.81 \\
\hline Emotional exhaustion month 1 & 2.79 & 0.86 & 2.60 & 0.79 & 1310 & $3.88^{* * *}$ \\
\hline Clinician-rated patient safety month 1 & 3.71 & 0.65 & 3.70 & 0.58 & 1307 & 0.42 \\
\hline Cognitive-behavioral teamwork month 4 & 5.22 & 0.80 & 5.28 & 0.74 & 935 & -1.27 \\
\hline Interpersonal teamwork month 4 & 3.14 & 0.63 & 3.13 & 0.63 & 927 & 0.002 \\
\hline Emotional exhaustion month 4 & 2.75 & 0.85 & 2.56 & 0.79 & 908 & $3.33^{* * *}$ \\
\hline Clinician-rated patient safety month 4 & 3.72 & 0.61 & 3.70 & 0.56 & 908 & 0.60 \\
\hline
\end{tabular}

Chi square: dichotomous variables; $t$ tests: continuous variables. Workload in hospital/ICU were not included in analyses because the number of clinicians who indicated their workload across all measurement occasions was insufficient

$M$ mean, $S D$ standard deviation, ICU intensive care unit

${ }^{* *} p<.01$ (two-tailed test); ${ }^{* * *} p<.001$ (two-tailed test)

\section{Clinician-rated patient safety}

We measured clinician-rated patient safety with a oneitem scale from the validated German, French, and Italian translations of the Hospital Survey of Patient Safety Culture (HSOPSC) [26-29]. Clinicians were asked to rate patient safety on their unit ("Please give your unit in this hospital an overall grade on patient safety"). Responses are given on a 5-point Likert Scale $(1=$ unsatisfactory, $5=$ excellent).
To examine how representative individual safety ratings are of general perception of safety in each unit, we measured the agreement on patient safety per unit by calculating within-group interrater reliability $\left(\mathrm{r}_{\mathrm{WG}}\right)$ values [30]. The $\mathrm{r}_{\mathrm{WG}}$ index compares the standard deviation (SD) of raters on each unit to the standard deviation that was to be expected if ratings were completely at random. The values ranged from .50 to .94 , with a mean of $.81(S D=.17)$, indicating that there was a high

Table 3 Correlations between study variables

\begin{tabular}{|c|c|c|c|c|c|c|c|c|c|c|c|c|c|c|}
\hline & & & 1 & 2 & 3 & 4 & 5 & 6 & 7 & 8 & 9 & 10 & 11 & 12 \\
\hline \multirow[t]{5}{*}{ Month 1} & 1 & Professional role & & & & & & & & & & & & \\
\hline & 2 & Cognitive-behavioral teamwork & $.18^{* * *}$ & $(.89)$ & & & & & & & & & & \\
\hline & 3 & Interpersonal teamwork & $.24^{* * *}$ & $.49^{* * *}$ & $(.86)$ & & & & & & & & & \\
\hline & 4 & Emotional exhaustion & .01 & $-.26^{* * *}$ & $-.23^{* * *}$ & $(.87)$ & & & & & & & & \\
\hline & 5 & Clinician-rated patient safety & $.14^{* * *}$ & $.49^{* * *}$ & $.38^{* * *}$ & $-.25^{* * *}$ & & & & & & & & \\
\hline \multirow[t]{4}{*}{ Month 4} & 6 & Cognitive-behavioral teamwork & $.14^{* * *}$ & $.69^{* * *}$ & $.45^{* * *}$ & $-.19^{* * *}$ & $.40^{* * *}$ & $(.89)$ & & & & & & \\
\hline & 7 & Interpersonal teamwork & $.21^{* * *}$ & $.48^{* * *}$ & $.66^{* * *}$ & $-.23^{* * *}$ & $.31^{* * *}$ & $.51^{* * *}$ & $(.87)$ & & & & & \\
\hline & 8 & Emotional exhaustion & .05 & $-.19^{* * *}$ & $-.20^{* * *}$ & $.81^{* * *}$ & $-.16^{* * *}$ & $-.22^{* * *}$ & $-.23^{* * *}$ & $(.88)$ & & & & \\
\hline & 9 & Clinician-rated patient safety & $.12^{* * *}$ & $.44^{* * *}$ & $.33^{* * *}$ & $-.18^{* * *}$ & $.56^{* * *}$ & $.46^{* * *}$ & $.35^{* * *}$ & $-.25^{* * *}$ & & & & \\
\hline \multirow[t]{4}{*}{ Month 7} & 10 & Cognitive-behavioral teamwork & $.17^{* * *}$ & $.71^{* * *}$ & $.43^{* * *}$ & $-.16^{* * *}$ & $.37^{* * *}$ & $.74^{* * *}$ & $.48^{* * *}$ & $-.13^{* * *}$ & $.41^{* * *}$ & $(.90)$ & & \\
\hline & 11 & Interpersonal teamwork & $.26^{* * *}$ & $.42^{* * *}$ & $.63^{* * *}$ & $-.16^{* * *}$ & $.21^{* * *}$ & $.45^{* * *}$ & $.68^{* * *}$ & $-.15^{* * *}$ & $.31^{* * *}$ & $.48^{* * *}$ & $(.89)$ & \\
\hline & 12 & Emotional exhaustion & .05 & $-.16^{* * *}$ & $-.23^{* * *}$ & $.75^{* * *}$ & $-.11^{*}$ & $-.13^{* *}$ & $-.13^{* * *}$ & $.83^{* * *}$ & $-.19^{* * *}$ & $-.22^{* * *}$ & $-.24^{* * *}$ & $(.88)$ \\
\hline & 13 & Clinician-rated patient safety & $.12^{*}$ & $.35^{* * *}$ & $.23^{* * *}$ & $-.18^{* * *}$ & $.48^{* * *}$ & $.42^{* * *}$ & $.33^{* * *}$ & $-.17^{* * *}$ & $.57^{* * *}$ & $.48^{* * *}$ & $.32^{* * *}$ & $-.18^{* * *}$ \\
\hline
\end{tabular}


level of agreement regarding overall safety between clinicians in each unit.

\section{Covariates}

Potential differences between professions in perceptions of teamwork, emotional exhaustion, and clinician-rated patient safety were taken into account by controlling for professional role (nurse/physician).

\section{Analyses}

\section{Hypothesis testing}

We tested our hypotheses by conducting structural equation modeling (SEM) analyses using Mplus version 7 [31]. This statistical method analyzes patterns of covariance (i.e., how much two or more variables change together) within a network of variables. To test causal relationships between variables, a cross-lagged design was used (see Fig. 1). Compared to cross-sectional data, which can only yield information on associations between variables at the same time and hence is mute about the causal direction, cross-lagged models take advantage of longitudinal data by estimating the effect of a predictor at an earlier time point on an outcome at a later time point.

Of particular importance, by simultaneously estimating the hypothesized causal effect (e.g., interpersonal teamwork at month 1 predicts emotional exhaustion at month 4; cross-lagged effect; oblique solid arrows in Fig. 1) as well as the alternative causal effect (e.g., emotional exhaustion at month 1 predicts interpersonal teamwork at month 4; reverse cross-lagged effect; oblique dashed arrows in Fig. 1), cross-lagged models inform us about the causal direction of the studied effects.

In addition to estimating the hypothesized crosslagged and reverse cross-lagged effects, the potentially confounding effect of each variable's temporal stability was taken into account by controlling for the baseline level of the variables across time (e.g., the effect of emotional exhaustion at month 1 on emotional exhaustion at month 4; autoregressive effect, horizontal arrows in Fig. 1).

Furthermore, the predictors at month 1 were correlated to account for shared variance among them (i.e., the extent to which variations in the predictors overlap; curved arrows in Fig. 1). To account for variance due to measurement occasion (i.e., variance that is not explained by the cross-lagged effects), we cross-sectionally correlated the residual variances at month 4 and month 7 (not depicted in Fig. 1 to increase readability).

Finally, we calculated the average of parallel effects between month 1 and month 4, and month 4 and month 7 (e.g., interpersonal teamwork at month 1 predicting emotional exhaustion at month 4 , and interpersonal teamwork at month 4 predicting emotional exhaustion at month 7). This approach identifies only stable effects as significant, and adjusts large effect sizes, thus reducing the complexity of the model and increasing precision and generalizability of results.

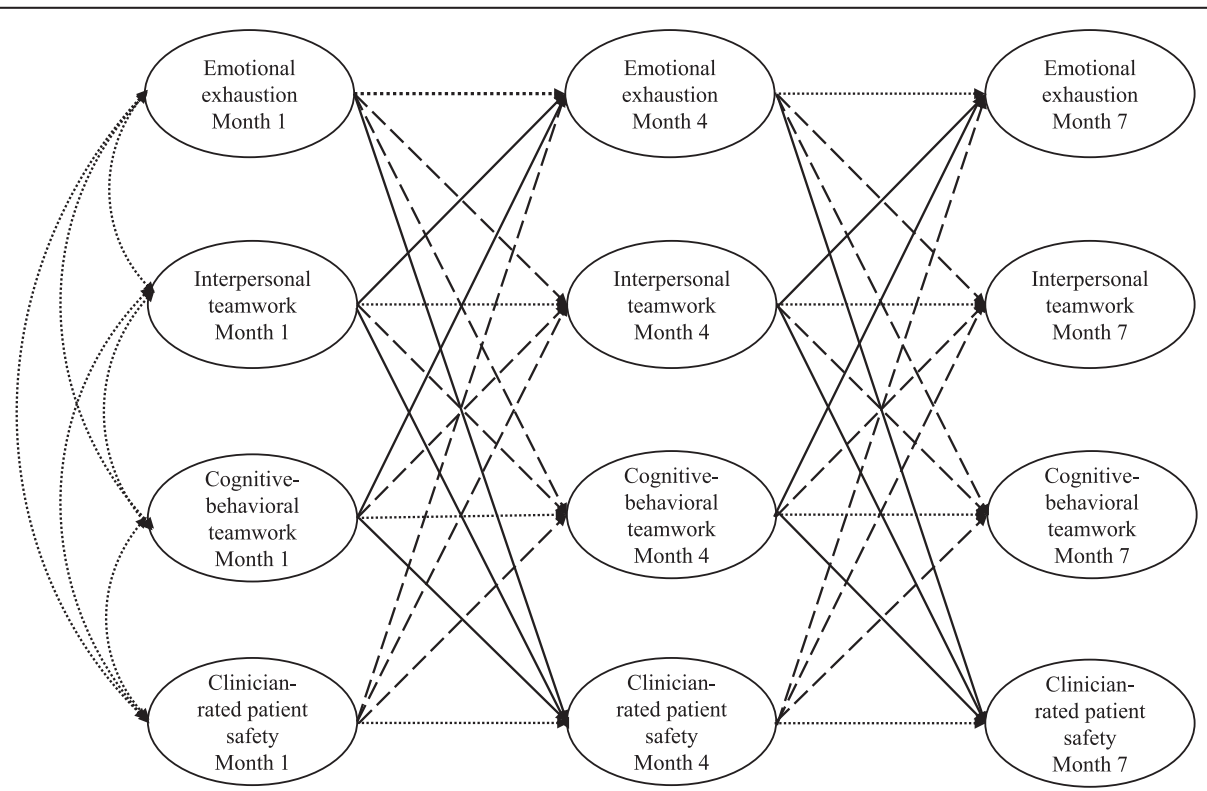

Fig. 1 Cross-lagged structural equation model testing longitudinal relationships between teamwork, clinician emotional exhaustion, and patient safety. Oblique solid arrows: hypothesized cross-lagged effects between measurement occasions. Oblique dashed arrows: reverse cross-lagged effects between measurement occasions. Horizontal arrows: auto-regressive paths of the same variable between different measurement occasions. Curved arrows: shared variance among the predictors 


\section{Model estimation and fit}

Full information maximum likelihood (FIML) estimation for complex survey data was applied. This approach incorporates all available data into the statistical analyses, thus including individuals who did not respond at all measurement occasions, or did not complete the survey at one measurement occasion. It yields more reliable results compared to traditional methods of handling missing data, such as pairwise or listwise deletion, which excludes individuals from analyses if their survey responses are incomplete and thus reduces statistical power.

We furthermore accounted for the clustered data structure: in this sample, clinicians are clustered within their teams. Data obtained from members of the same team are not independent [32]. Ignoring clustered data structures can lead to underestimation of standard errors of effects and thus an overestimation of statistical significance. Data clustering was taken into account by adjusting the standard error [33].

\section{Results}

Based on an outlier analysis following best-practice recommendations we deleted three ICUs with a participation of less than five people per unit from the sample [34]. Descriptive statistics and correlations are reported in Tables 1 and 3, respectively.

\section{Longitudinal relationships between teamwork, emotional exhaustion, and clinician-rated patient safety}

Figure 2 shows the significant cross-lagged and reverse cross-lagged effects of the structural equation model. Analyses revealed that cognitive-behavioral teamwork, interpersonal teamwork, emotional exhaustion, and clinician-rated patient safety were interrelated. Cognitivebehavioral teamwork $(\beta=.17, p=.03)$, but not interpersonal teamwork $(\beta=.03, p=.30)$ predicted an increase in clinician-rated patient safety (see Table 4). In turn, clinicians' safety perceptions predicted an increase in cognitive-behavioral teamwork ( $\beta=.08, p=.03$ ).

Moreover, there was a reciprocal lagged relationship of cognitive-behavioral teamwork on interpersonal teamwork $(\beta=.13, p=.03)$ and vice versa $(\beta=.09, p=.03)$. Thus, cognitive-behavioral teamwork predicts an improvement in interpersonal teamwork and interpersonal teamwork predicts an improvement in cognitivebehavioral teamwork.

Cognitive-behavioral teamwork $(\beta=-.01, p=.02)$ and interpersonal teamwork $(\beta=-.03, p=.02)$ had no effect on later emotional exhaustion. However, emotional exhaustion predicted a deterioration of the quality of interpersonal teamwork ( $\beta=-.07, p=.02)$. In addition, there was a tendency for emotional exhaustion to predict a decrease in clinician-rated patient safety $(\beta=-.05, p=.09)$.
In general, physicians reported better cognitivebehavioral teamwork $(\beta=.08, p=.02)$, interpersonal teamwork $(\beta=.15, p=.02)$, clinician-rated patient safety $(\beta=.06, p=.02)$, and higher emotional exhaustion $(\beta=.04$, $p=.01)$ than nurses.

\section{Discussion}

This study highlights the importance of longitudinal research approaches to examine the complex causal interrelations between teamwork, clinician emotional exhaustion, and patient safety in intensive care settings. Overall, our results suggest that emotionally exhausted clinicians are less able to contribute to effective teamwork, which in turn is necessary to maintain patient safety. Specifically, our analyses showed that low clinician emotional exhaustion increased the quality of interpersonal teamwork. Interpersonal teamwork had a positive effect on cognitive-behavioral teamwork and vice versa. Finally, cognitive-behavioral teamwork positively affected clinician-rated patient safety.

The current study goes beyond prior research that tended to focus on isolated aspects of the multidimensional construct of teamwork [35]. Generally, previous cross-sectional studies addressed team cognitions and behaviors in relation to patient safety, and interpersonal teamwork aspects in association with clinician emotional exhaustion or burnout in general $[35,36]$. We investigated the effect of cognitive-behavioral and interpersonal teamwork on emotional exhaustion and patient safety.

\section{Background}

Based on the job demands-resources model, we assumed that effective teamwork may act as a job resource (i.e., aspects of a job that can buffer work demands, achieve work goals, and foster employee well-being) that positively affects patient safety [37].

According to the conservation of resources theory's (COR) gain cycles, individuals or teams who initially possess plenty of resources are more likely to gain additional resources [38]. Positive interpersonal teamwork may act as a resource fostering the exchange of safetyrelevant information between professions, thus increasing patient safety. Similarly, positive cognitive-behavioral teamwork may act as a resource that leads to procedures being carried out more smoothly, fewer errors, and thus higher patient safety. Effective cognitive-behavioral teamwork may be fostered by a good safety climate, which has been shown to be associated with fewer self-reported errors [39]. Teamwork as a resource may also buffer the impact of daily stressors and thus prevent clinician emotional exhaustion.

Lastly, we expected clinician emotional exhaustion to have a negative effect on patient safety. Emotional exhaustion can develop in individuals whose resources are insufficient to 


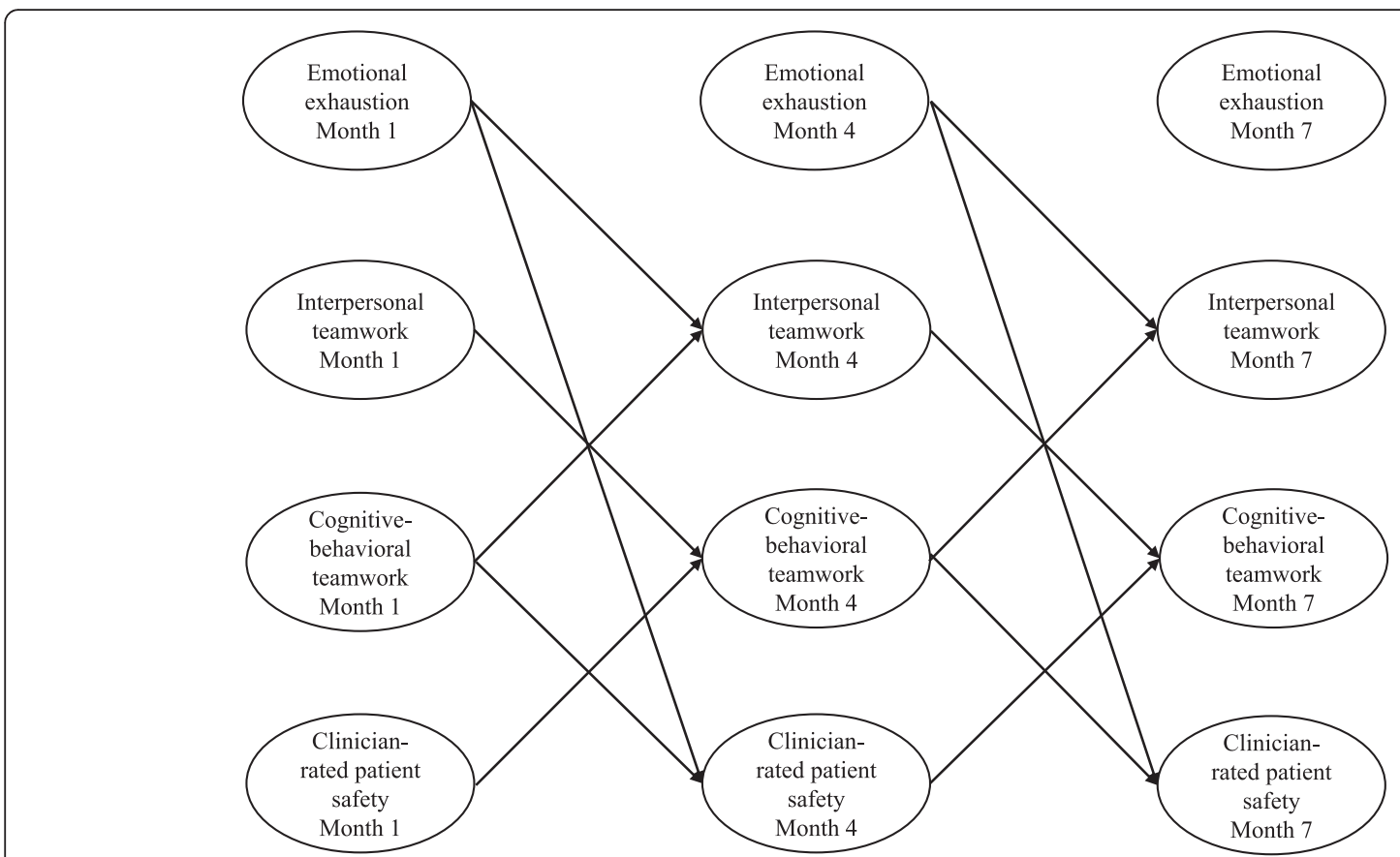

Fig. 2 Cross-lagged structural equation model showing significant (reverse) cross-lagged effects (statistics reported in Table 4)

meet the cognitive, emotional, or physical demands of their job [39, 40]. Emotionally exhausted clinicians are less vigilant; their motivation to exhibit safe work practices may decrease; and thus errors are more likely to occur $[41,42]$.

\section{Differential associations of interpersonal and cognitive- behavioral teamwork with clinician emotional exhaustion and clinician-rated patient safety}

Our results showed that cognitive-behavioral teamwork had a positive effect on clinician-rated patient safety, but it was not related to clinician emotional exhaustion. Tangible cognitive-behavioral team processes are instrumental to complete patient care-related tasks, such as pooling collective expertise to solve problems. These processes contribute to higher patient safety, and clinicians may be required to participate in such team processes despite being emotionally exhausted. However, they are not required to invest resources into interpersonal relationships, which might explain the finding that interpersonal teamwork suffered as a consequence of high clinician emotional exhaustion.

Interpersonal teamwork, a global evaluation of the quality of interprofessional collaboration, had no immediate impact on clinician-rated patient safety. It did, however, facilitate cognitive-behavioral teamwork, which in turn positively affected clinician-rated patient safety.

The results highlight the importance of longitudinal studies that go beyond mere cross-sectional associations between teamwork, patient safety and emotional exhaustion, but actually test assumptions concerning causal directions between these constructs.

Table 4 Standardized estimates of the structural coefficients in the model

\begin{tabular}{|c|c|c|c|c|}
\hline & \multicolumn{4}{|l|}{ Outcome } \\
\hline & Cognitive-behavioral teamwork & Interpersonal teamwork & Emotional exhaustion & Clinician-rated patient safety \\
\hline \multicolumn{5}{|l|}{ Predictor } \\
\hline Professional role & $.08^{* * *}(.02)$ & $.15^{* * *}(.02)$ & $.04^{*}(.01)$ & $.06^{* *}(.02)$ \\
\hline Cognitive-behavioral teamwork & $.63^{* * *}(.03)$ & $.13^{* * *}(.03)$ & $-.01(.02)$ & $.17^{* * *}(.03)$ \\
\hline Interpersonal teamwork & $.09^{* *}(.03)$ & $.56^{* * *}(.32)$ & $-.03(.02)$ & $.03(.30)$ \\
\hline Emotional exhaustion & $-.01(.02)$ & $-.07^{* *}(.02)$ & $.82^{* * *}(.01)$ & $-.05(.09)$ \\
\hline Clinician-rated patient safety & $.08^{* *}(.03)$ & $-.01(.02)$ & $.02(.02)$ & $.50^{* * *}(.03)$ \\
\hline
\end{tabular}

Estimates were constrained to be equal across time (e.g., effect of interpersonal teamwork at month 1 on emotional exhaustion at month 4 was set to be equal to the effect of interpersonal teamwork at month 4 on emotional exhaustion at month 7) to increase the reliability and validity of the estimates. Standard errors are in brackets. Model fit indices: RMSEA (root mean square error of approximation) $=0.05$, CFI (comparative fit index) $=0.96$, TLI (Tucker-Lewis Index) $=0.93$, indicating a good fit $[48,49]$

${ }^{*} p<.05$ (two-tailed test); ${ }^{* *} p<.01$ (two-tailed test); ${ }^{* * *} p<.001$ (two-tailed test) 


\section{Reciprocal relationships between interpersonal and cognitive-behavioral teamwork}

Furthermore, our findings demonstrate that the interpersonal and cognitive-behavioral dimensions of teamwork are mutually dependent: On the one hand, better teamwork between professions facilitates cognitive-behavioral teamwork, such as coordination, communication, and cognitive functioning. This is in line with previous work suggesting that interpersonal teamwork forms the foundation on which cognitive-behavioral teamwork components are executed [2]. Trust and mutual respect foster a positive team climate that encourages individuals to contribute their expertise to the common goal, to speak up and voice their concerns in situations where they might deviate from the majority, or to report errors [42]. On the other hand, effective cognitive-behavioral teamwork improves the interpersonal quality of teamwork between professions.

\section{Effects of emotional exhaustion on patient safety}

Our analyses showed that clinician emotional exhaustion and patient safety do not evolve independently. First, our results suggest that emotional exhaustion may have a direct effect on clinician-rated patient safety. These findings complement a previous cross-sectional study conducted in intensive care units showing that emotional exhaustion was not only related to clinician-rated patient safety, but also to standardized mortality ratios [43].

Second, our results imply that clinician emotional exhaustion and clinician-rated patient safety are connected via the reciprocal relationships between interpersonal teamwork and safety organizing: clinicians with low emotional exhaustion may possess more resources to invest in interprofessional relationships. These, in turn, facilitate cognitive-behavioral teamwork and vice versa. Finally, cognitive-behavioral teamwork contributes to higher clinician-rated patient safety.

\section{Interprofessionalism}

Finally, our results highlight the importance of including multiple professions when investigating teamwork. We confirmed that nurses' and physicians' ratings of teamwork, emotional exhaustion, and clinician-rated patient safety differ. Previous survey studies that investigated relationships between teamwork and clinician emotional exhaustion or patient safety rarely included multiple professions or explicitly addressed interprofessional teamwork, particularly in intensive care settings [44]. However, interprofessionalism is a defining feature of teams. Our study shows that even in highly technical environments, such as intensive care, the quality of interprofessional collaboration is an important aspect of teamwork that complements the cognitive-behavioral dimension.

\section{Limitations}

The results of this study should be interpreted with some limitations in mind. We focused on the emotional exhaustion subscale from the Maslach Burnout Inventory because the sample size on the unit level did not allow for testing a more complex model that included the depersonalization and reduced personal accomplishment subscales [24]. Nevertheless, we believe that our results are representative and reliable. Emotional exhaustion is the core dimension of burnout and the most reliable and valid subscale across languages and cultures [45]. Moreover, 55 out of 81 Swiss ICUs and a total of 2100 clinicians constitute a high participation rate and large sample size at the individual level.

For economic reasons, patient safety was measured with a single-item indicator that assessed clinicians' perceptions of overall safety in their unit and may therefore be less reliable than detailed surveys or objective indicators. However, previous research has shown that subjective safety ratings are indicative of objective patient safety such as standardized mortality ratios, as subjective and objective safety measures partly overlap [46]. In addition, our data show a high level of agreement regarding patient safety between team members. This illustrates that safety perceptions are a unit attribute and not only an individual rating impacted by emotional exhaustion and associated negative cognitions. In this study, we focused on developing a general understanding of the causal relationships between two aspects of teamwork, emotional exhaustion, and patient safety. Future research might include a multi multifaceted faceted conceptualization of patient safety into this model.

\section{Practical implications}

Interpersonal and cognitive-behavioral aspects of teamwork build upon one another and are thus both important for effective team functioning. Even in hightechnology environments such as the ICU setting, good interpersonal relationships can facilitate cognitivebehavioral teamwork. Thus, interventions targeting teamwork should be designed with both teamwork aspects in mind, as such interventions carry the potential to reinforce each other: inclusion of the entire, multiprofessional team; focusing on similarities and shared goals; building of shared mental models; and improving communication and coordination.

Observational studies in intensive care settings have shown the significance of cognitive-behavioral teamwork for immediate team performance outcomes [2]. Our study complements these findings by highlighting longterm effects. Long-term investment in teamwork is likely to build routine on which team members can rely in stressful situations. Previous research has shown that burnout (including emotional exhaustion) can spread 
from one intensive care clinician to another [47]. It is thus important to prevent the development of clinician emotional exhaustion before it becomes a problem for the entire team, as emotionally exhausted clinicians are less likely to possess the resources to engage in or benefit from team trainings.

\section{Conclusions}

To our knowledge, this is the first study to investigate simultaneous relationships between teamwork, clinician emotional exhaustion, and clinician-rated patient safety using an interprofessional sample. Our results highlight the importance of longitudinal studies, which are necessary to detect long-term, causal effects. Targeting clinician emotional exhaustion is essential in order to ensure effective teamwork and a high level of patient safety. Interventions intended to reduce clinician emotional exhaustion may set a cycle in motion that increases patient safety via mutual reinforcement of interpersonal and cognitive-behavioral teamwork.

\section{Ethics approval and consent to participate}

Ethics permission was granted from the university and the cantonal ethics committees $(75,2013-06-03$; 024/13-CER-FR, 2013-24-06). Written consent to participate was obtained per unit from the unit leaders. Upon accessing the online survey, participants were asked for their consent to participate, and assured complete anonymity and confidential handling of their data.

\section{Endnotes}

${ }^{1}$ The survey also included the depersonalization and personal accomplishment scales of the Maslach Burnout Inventory [24]. The number of relationships between variables defined in a clustered SEM is limited by the sample size at the unit level. Based on our core research aim of testing simultaneous interrelations and the results of a previous cross-sectional study, which showed that emotional exhaustion was the main predictor of both clinician-rated and objective indicators of patient safety, we opted to exclude the other burnout components in the main analyses to develop a meaningful and reliable statistical model [44].

\section{Abbreviations}

CFI: comparative fit index; COR: Conservation of Resources theory; FIML: full information maximum likelihood; HSOPSC: Hospital Survey of Patient Safety Culture; ICU: intensive care unit; M: mean; MBI-HSS: Maslach Burnout Inventory - Human Services Survey; NWI-R: Nursing Work Index - revised; RMSEA: root mean square error of approximation; RN: registered nurse; $r_{\text {WG: }}$ within-group interrater reliability; SD: standard deviation; SEM: structural equation modeling; TLI: Tucker-Lewis Index.

\section{Competing interests}

The authors declare that they have no competing interests.

\section{Authors' contributions}

AW designed the study, collected the data, analyzed and interpreted the data, and drafted the manuscript. LM substantially contributed to data analysis and interpretation of results, and helped draft the manuscript. TM substantially contributed to the design of the study, interpretation of results, and drafting of the manuscript. All authors read and approved the final manuscript.

\section{Acknowledgements}

This study was conducted in collaboration with the Swiss Society for Intensive Care Medicine (SGI). We thank the local study coordinators of the participating ICUs for their help with the implementation of this study and with data collection, and the staff on each unit for answering our questionnaire.

We would furthermore like to thank Mariel Dardel and Nicola Rudolph for their help with coordinating the data collection.

\section{Funding}

This project was part of the Swiss National Science Foundation grant number PP00P1_128616 "Teamwork in acute patient care: how team processes and clinicians' perceptions of teamwork affect patient safety", which supported Annalena Welp and Tanja Manser. Laurenz L. Meier was supported by the Swiss National Science Foundation grant number PZOOP1-142393.

\section{Author details}

${ }^{1}$ Department of Psychology, University of Fribourg, Rue Faucigny 2, 1700 Fribourg, Switzerland. ${ }^{2}$ Institute of Work and Organizational Psychology, University of Neuchâtel, Neuchâtel, Switzerland. ${ }^{3}$ Institute for Patient Safety, University Hospital Bonn, Stiftsplatz 12, 53111 Bonn, Germany. ${ }^{4}$ ETH Zurich, Department of Management, Technology and Economics, Weinbergstrasse 56/58, 8092 Zurich, Switzerland.

Received: 22 October 2015 Accepted: 1 April 2016

Published online: 19 April 2016

References

1. Dietz AS, Pronovost PJ, Mendez-Tellez PA, Wyskiel R, Marsteller JA, Thompson DA, et al. A systematic review of teamwork in the intensive care unit: what do we know about teamwork, team tasks, and improvement strategies? J Crit Care. 2014;29:908-14.

2. Marks MA, Mathieu JE, Zaccaro SJ. A temporally based framework and taxonomy of team processes. Acad Manage Rev. 2001:26:356-76.

3. Dickinson TL, McIntyre RM. A conceptual framework for teamwork measurement. In: Brannick MT, Salas E, Prince C, editors. Team performance assessment and measurement: theory, methods, and applications. Mahwah: Lawrence Erlbaum Associates; 1997. p. 19-43.

4. Salas E, Cooke NJ, Rosen MA. On teams, teamwork, and team performance: discoveries and developments. Hum Factors. 2008:50:540-7.

5. Martin JS, Ummenhofer W, Manser T, Spirig R. Interprofessional collaboration among nurses and physicians: making a difference in patient outcome. Swiss Med Wkly. 2010;140:w13062.

6. Baggs J, Schmitt M, Mushlin A, Mitchell P, Eldrege D, Oakes D. Association between nurse-physician collaboration and patient outcomes in three intensive care units. Crit Care Med. 1999;27:1991-8.

7. McCulloch P, Mishra A, Handa A, Dale T, Hirst G, Catchpole K. The effects of aviation-style non-technical skills training on technical performance and outcome in the operating theatre. Qual Safe Health Care. 2009:18:109-15.

8. Burtscher MJ, Kolbe M, Wacker J, Manser T. Interactions of team mental models and monitoring behaviors predict team performance in simulated anesthesia inductions. J Exp Psychol Appl. 2011;17:257-69.

9. Sutinen R, Kivimaki M, Elovainio M, Forma P. Associations between stress at work and attitudes towards retirement in hospital physicians. Work Stress. 2005;19:177-85

10. Van Bogaert P, Clarke S, Roelant E, Meulemans H, Van de Heyning P. Impacts of unit-level nurse practice environment and burnout on nurse-reported outcomes: a multilevel modelling approach. J Clin Nurs. 2010;19:1664-74.

11. Teixeira C, Ribeiro O, Fonseca A, Carvalho A. Burnout in intensive care units - a consideration of the possible prevalence and frequency of 
new risk factors: a descriptive correlational multicentre study. BMC Anesthesiol. 2013;13:38.

12. Rothschild JM, Landrigan CP, Cronin JW, Kaushal R, Lockley SW, Burdick E, et al. The Critical Care Safety Study: the incidence and nature of adverse events and serious medical errors in intensive care. Crit Care Med. 2005:33:1694-700

13. Merlani P, Verdon M, Businger A, Domenighetti G, Pargger H, Ricou B. Burnout in ICU caregivers: a multicenter study of factors associated to centers. Am J Respir Crit Care Med. 2011;184:1140-6.

14. Kanai-Pak M, Aiken LH, Sloane DM, Poghosyan L. Poor work environments and nurse inexperience are associated with burnout, job dissatisfaction and quality deficits in Japanese hospitals. J Clin Nurs. 2008;17:3324-9.

15. Klopper HC, Coetzee SK, Pretorius R, Bester P. Practice environment, job satisfaction and burnout of critical care nurses in South Africa. J Nurs Manage. 2012;20:685-95.

16. Vogus TJ, Sutcliffe KM. The Safety Organizing Scale: development and validation of a behavioral measure of safety culture in hospital nursing units. Med Care. 2007;45:46-54.

17. Ausserhofer D, Schubert M, Blegen M, De Geest S, Schwendimann R. Validity and reliability on three European language versions of the Safety Organizing Scale. Int J Qual Health Care. 2013;25:157-66.

18. Lake ET. Development of the practice environment scale of the nursing work index. Res Nurs Health. 2002;25:176-88.

19. Sermeus W, Aiken LH, Van den Heede K, Rafferty AM, Griffiths P, Moreno-Casbas MT, et al. Nurse forecasting in Europe (RN4CAST): rationale, design and methodology. BMC Nurs. 2011;10:6.

20. Ausserhofer D, Desmedt M, Schubert M, Schwendimann R, De Geest S. RN4Cast - Schweizer Ergebnisse der weltweit grössten Outcome-Studie in der Pflege. Pflegenetz. 2012;2:4-7.

21. Büssing A, Glaser J. Managerial stress und burnout. A collaborative international study (CISMS). Die deutsche Untersuchung. München: Technische Universität, Lehrstuhl für Psychologie; 1998.

22. Dion G, Tessier R. Validation de la traduction de l'Inventaire d'épuisement professionnel de Maslach et Jackson. Can J Behav Sci. 1994;26:210-27.

23. Pisanti R, Lombardo C, Lucidi F, Violani C, Lazzari D. Psychometric properties of the Maslach Burnout Inventory for Human Services among Italian nurses: a test of alternative models. J Adv Nurs. 2013;69:697-707.

24. Maslach C, Jackson SE, Leiter MP. Maslach Burnout Inventory manual. Palo Alto: Consulting Psychologists Press; 1996.

25. Maslach C, Schaufeli W, Leiter M. Job burnout. Annu Rev Psychol. 2001;52:397-422

26. Sorra J, Nieva VF. Psychometric analysis of the Hospital Survey on Patient Safety. Final report to Agency for Health Care Research and Quality. Washington: AHRQ; 2004

27. Pfeiffer $Y$, Manser T. Development of the German version of the Hospital Survey on Patient Safety Culture: dimensionality and psychometric properties. Saf Sci. 2010;48:1452-62.

28. Occelli P, Quenon J-L, Kret M, Domeca S, Delaperche F, Claverie O, et al. Validation of the French version of the Hospital Survey on Patient Safety Culture questionnaire. Int J Qual Health Care. 2013;25:459-68.

29. Bagnasco A, Tibaldi L, Chirone P, Chiaranda C, Panzone MS, Tangolo D, et al. Patient safety culture: an Italian experience. J Clin Nurs. 2011;20:1188-95.

30. James $L R$, Demaree $R G$, Wolf $G$. Estimating within-group interrater reliability with and without response bias. J Appl Psychol. 1984;69:85-98.

31. Muthén LK, Muthén BO. MPLUS (Version 7). [Computer software]. Los Angeles, CA: Muthén \& Muthén; 2012.

32. Hayes AF. A primer on multilevel modeling. Hum Commun Res. 2006;32:385-410.

33. Muthén LK, Muthén BO. Mplus user's guide. 7th ed. Los Angeles, CA: Muthén \& Muthén; 2012.

34. Aguinis $\mathrm{H}$, Gottfredson RK, Joo H. Best-practice recommendations for defining, identifying, and handling outliers. Organ Res Methods. 2013;16:270-301.

35. Budge C, Carryer J, Wood S. Health correlates of autonomy, control and professional relationships in the nursing work environment. J Adv Nurs. 2003:42:260-8

36. Rathert C, Williams ES, Lawrence ER, Halbesleben JRB. Emotional exhaustion and workarounds in acute care: cross sectional tests of a theoretical framework. Int J Nurs Stud. 2012;49:969-77.

37. Demerouti E, Bakker AB, Nachreiner F, Schaufeli WB. The job demandsresources model of burnout. J Appl Psychol. 2001;86:499-512.
38. Hobfoll SE. The influence of culture, community, and the nested-self in the stress process: advancing conservation of resources theory. Appl Psychol. 2001;50:337-21.

39. Valentin A, Schiffinger M, Steyrer J, Huber C, Strunk G. Safety climate reduces medication and dislodgement errors in routine intensive care practice. Intensive Care Med. 2013;39:391-8.

40. Vuori M, Akila R, Kalakoski V, Pentti J, Kivimaki M, Vahtera J, et al. Association between exposure to work stressors and cognitive performance. J Occup Environ Med. 2014:56:354-60.

41. Halbesleben JRB, Rathert C. Linking physician burnout and patient outcomes: Exploring the dyadic relationship between physicians and patients. Health Care Manage R. 2008;33:29-39.

42. Nembhard IM, Edmondson AC. Making it safe: the effects of leader inclusiveness and professional status on psychological safety and improvement efforts in health care teams. J Organ Behav. 2006:27:941-66.

43. Welp A, Meier LL, Manser T. Emotional exhaustion and workload predict clinician-rated and objective patient safety. Front Psychol. 2015;5:1573.

44. Thomas E, Sexton J, Helmreich R. Discrepant attitudes about teamwork among critical care nurses and physicians. Crit Care Med. 2003;31:956-9.

45. Poghosyan $L$, Aiken $L H$, Sloane DM. Factor structure of the Maslach Burnout Inventory: an analysis of data from large scale cross-sectional surveys of nurses from eight countries. Int J Nurs Stud. 2009:46:894-902.

46. Lawton R, O'Hara JK, Sheard L, Reynolds C, Cocks K, Armitage G, et al. Can staff and patient perspectives on hospital safety predict harm-free care? An analysis of staff and patient survey data and routinely collected outcomes. BMJ Qual Saf. 2015;24:369-76.

47. Bakker A, Le Blanc P, Schaufeli W. Burnout contagion among intensive care nurses. J Adv Nurs. 2005;51:276-87.

48. Hu LT, Bentler PM. Cutoff criteria for fit indexes in covariance structure analysis: Conventional criteria versus new alternatives. Struct Equ Modeling. 1999;6:1-55.

49. MacCallum RC, Austin JT. Applications of structural equation modeling in psychological research. Annu Rev Clin Psychol. 2000;51:201-26.

\section{Submit your next manuscript to BioMed Central and we will help you at every step:}

- We accept pre-submission inquiries

- Our selector tool helps you to find the most relevant journal

- We provide round the clock customer support

- Convenient online submission

- Thorough peer review

- Inclusion in PubMed and all major indexing services

- Maximum visibility for your research

Submit your manuscript at www.biomedcentral.com/submit
Biomed Central 